\title{
Original
}

\section{Tamizaje de Diabetes Mellitus tipo 2 en atención primaria}

\author{
Adriana Laclé ', Carmen Peralta ${ }^{2}$
}

\section{Resumen}

Introducción: Actualmente en Costa Rica debería considerarse la Diabetes Mellitus tipo 2, un problema de salud pública, por el impacto de sus complicaciones crónicas tanto en el ámbito individual y familiar, como en lo económico, en la escala nacional. Su detección y tratamiento temprano ha demostrado que reduce la carga de sus complicaciones, por lo que su tamizaje podría ser oportuno en personas que tengan factores de riesgo asociados. El objetivo de este estudio fue valorar los resultados de la implementación de un tamizaje de la diabetes tipo 2 a nivel de atención primaria, en personas en riesgo de desarrollarla.

Metodología: El tamizaje se efectuó durante el año 2000, en la población adulta con al menos un factor de riesgo asociado, en 6 Equipos Básicos de Atención Integral en Salud (EBAIS), del Área de Salud 3 de Desamparados. Este se incorporó en el quehacer diario del EBAIS, sin alterar su programación. Se utilizaron cuatro estrategias para la realización del tamizaje: la oportunista, la selectiva, las programadas a grupos cautivos u organizacionales, y las jornadas de atención comunal o nacional.

Resultados: Durante 2000 se lograron captar 174 diabéticos, diagnosticados por primera vez, o que ya lo eran, pero que no estaban registrados al carecer de control en el EBAIS, aunque vivían en el área. La estrategia predominante fue la oportunista, cuya responsabilidad recayó principalmente en el médico. La estrategia programada se realizó por medio de "jornadas" durante las ferias de salud en cada EBAIS y en el Día Mundial de la Diabetes, como parte de la jornada nacional; la cautiva se llevó a cabo en el Hogar Diurno de Ancianos. La selectiva, mediante visita domiciliar, fue mínima, por falta de glucómetros. Estas estrategias permitieron que la cobertura de captación aumentara de un $50 \%$ a finales de 1999 a un $70.2 \%$ a finales de 2000, considerando la cobertura como el porcentaje dado por el número de pacientes diabéticos captados o registrados en cada EBAIS, entre el número de personas diabéticas estimadas para

Instituto de Investigaciones en Salud, Universidad de Costa Rica.

2 Área 3 de Desamparados, C.C.S.S.

Abreviaturas: DM2, Diabetes Mellitus tipo 2; PRDM, personas de alto riesgo de desarrollar;ATAP, Asistente de Atención Primaria;ASIS, Análisis de Situación de Salud; EBAIS, Equipo Básico de Atención Integral en Salud; HbAlc, hemoglobina glicosilada.

Correspondencia: Dra. Adriana Laclé, alacle@cariari.ucr.ac.cr

ISSN 0001-6002/2006/48/1/17-23 Acta Médica Costarricense, @2006

Colegio de Médicos y Cirujanos cada EBAIS (considerando su prevalencia igual al 5\% de la población de 20 y más años).

Conclusión: Costa Rica puede lograr la detección temprana y tratamiento oportuno de la Diabetes Mellitus tipo 2, por medio de un tamizaje en subgrupos poblacionales de alto riesgo. El esfuerzo ya se está haciendo con la estrategia oportunista. Este estudio demuestra que esta no solo se puede implementar efectivamente, sino que, además, puede realizarse con muy poco esfuerzo. Ahora bien, la organización del Sistema de Salud con base en la Atención Primaria, permitiría desarrollar otra de las estrategias de tamizaje para obtener una mayor cobertura sin alto costo, es decir, el tamizaje domiciliar. Logísticamente existen las condiciones para realizarlo; lo que se necesita es llevar a cabo un estudio comunitario controlado para ver su costobeneficio. Si el resultado de este estudio es favorable, se podría implementar en toda Costa Rica como parte de la atención integral de la Diabetes Mellitus tipo 2, convirtiendo al país en pionero en el mundo en esta estrategia.

Descriptores: Diabetes Mellitus tipo 2, tamizaje, detección temprana

Key words: Diabetes Mellitus type 2, early detection, screening

Recibido: 6 de octubre de 2005

Aceptado: 8 de noviembre de 2005 
La Diabetes Mellitus tipo 2 (DM2) es actualmente considerada como una patología que se presenta en forma de "epidemia" en la mayoría de los países y en especial en los del tercer mundo. Costa Rica no escapa de esta tendencia, sobre todo si se considera el envejecimiento rápido de su población ${ }^{2}$. Nuestro perfil epidemiológico ha variado en menos de 50 años, de uno predominantemente de enfermedades infectocontagiosas a otro en donde las enfermedades crónicas son las más prevalentes, entre ellas, la DM2, tanto en relación con la morbilidad como con la mortalidad.

No existen estudios de prevalencia nacional, solo de prevalencia autoreferida ${ }^{3}$, pero se estima que entre un $5 \%$ y un $6 \%$ de la población de 20 y más años tiene diabetes, ascendiendo esta cifra para 2000, según población del último censo, a aproximadamente 134,000 habitantes.

En 1998, la Caja Costarricense de Seguro Social (CCSS), que cubre en atención en salud al 95\% de la población, contaba con 66.000 diabéticos (as) en tratamiento, bien con hipoglicemiantes orales o con insulina. Esto hace suponer que un porcentaje importante de ellos no están en control o desconocen que son diabéticos. En otros países también se presenta este fenómeno, como en Estados Unidos y Canadá, en donde se considera que por cada paciente diagnosticado existe uno que no lo estát, ${ }^{4}$. No sabemos cuántas personas diabéticas tipo 2 hay exactamente en Costa Rica.

El aumento esperado en la prevalencia de la DM2 en años futuros, aunado a que es la patología que mayor costo genera en la atención hospitalaria y la segunda causa de consulta externa en el adulto, permite afirmar que la DM2 es un problema de Salud Pública en Costa Rica ${ }^{3}$.

El inicio de esta enfermedad es usualmente asintomático en sus estadios tempranos, por lo que se mantiene sin diagnóstico por varios años. No obstante, los niveles de hiperglicemia en esos años pueden afectar los órganos blandos de la DM2, sobre todo los ojos ${ }^{6-8}$, riñones $^{9-10}$, corazón, nervios y vasos sanguíneos de las extremidades inferiores ${ }^{11,12}$. Estudios epidemiológicos demuestran que estos órganos se encuentran dañados en un porcentaje alto, varios años antes del diagnóstico clínico ${ }^{13,14}$.

La detección temprana y el tratamiento oportuno ha demostrado reducir la carga de las complicaciones de la DM2, por lo que el tamizaje para su detección temprana debería considerarse. Sin embargo, el tamizaje debe realizarse bajo ciertas condiciones, ya que la probabilidad de identificar a un individuo asintomático con diabetes, en la población general con un tamizaje al azar es pequeña; pero realizada en un grupo de alto riesgo, es mayor ${ }^{15,16}$.

Algunos subgrupos específicos de personas tienen una prevalencia más alta de la enfermedad que la población como un todo; entre ellos, los mayores de 45 años, con obesidad, con hipertensión arterial, con dislipidemia, con antecedentes heredo familiar de DM2, con antecedentes de diabetes gestacional, de macrosomía y con intolerancia a la glucosa ${ }^{17,18}$. Los factores de riesgo más importantes para desarrollar DM2 se resumen en el Cuadro $1^{17}$.

Faltan estudios de alta calidad sobre el costo-beneficio del uso del tamizaje de DM2 en personas con uno o más factores de riesgo para desarrollarla (PRDM) ${ }^{18}$; no obstante, podemos afirmar que el primer paso hacia una atención efectiva de esta enfermedad es su diagnóstico temprano.

Se propuso en esta investigación en servicio, valorar la implementación de 4 estrategias de tamizaje de DM2 para su detección temprana en PRDM del Área de Salud 3 de Desamparados, incorporando el tamizaje en el quehacer diario del EBAIS, sin alterar su programación. El objetivo final fue aumentar el porcentaje de cobertura de diabéticos en control o conocidos por el asistente de atención primaria (ATAP). Además, se quiso conocer la calidad del sistema de registro de los diabéticos en atención primaria, en los EBAIS estudiados.

\section{Metodología}

Población: Personas de $\geq 20$ años y DM2 captadas según el Análisis de Situación de Salud (ASIS) de 1999, de 6 EBAIS del Área de Salud 3 de Desamparados: Patarrá, Fátima, Guidos 2, Guidos 4, Río Azul y Linda Vista (Cuadro 2).

\section{Materiales y métodos}

I. Se propuso al equipo de salud de los 6 EBAIS realizar un tamizaje de DM2 por medio de 4 estrategias, dentro de su programación de atención integral durante el año 2000 .

A. Estrategias para el tamizaje. Se seleccionaron 4 tipos: la oportunista, como parte de la atención integral de todo individuo que consultó al médico en el EBAIS por cualquier patología; 2 tipos de estrategia programada, una a grupos cautivos (ej: Hogar de Ancianos, fábricas) y otra a grupos organizados o jornadas, y la selectiva, por medio de la visita domiciliar de rutina y de riesgo (familia en riesgo) por los ATAPS.

Durante 2000, la estrategia que predominó fue la "oportunista", recayendo la responsabilidad especialmente en el médico. La única estrategia programada a grupos cautivos se realizó en el Hogar Diurno de Río Azul. El otro tipo de estrategia programada se efectuó por medio de "jor- 


\begin{tabular}{|c|}
\hline $\begin{array}{c}\text { Cuadro 1. Factores de riesgo para Diabetes } \\
\text { Mellitus según la Asociación Diabética } \\
\text { Americana }\end{array}$ \\
\hline $\begin{array}{l}\text { Historia familiar de DM (padres o hermanos con } \\
\text { diabetes) }\end{array}$ \\
\hline Obesidad ( $\geq 20 \%$ peso ideal o IMC $\geq 27 \mathrm{~kg} / \mathrm{m}^{2}$ ) \\
\hline $\begin{array}{l}\text { Raza /etnia (eg; africoamericanos, hispanoamericanos, } \\
\text { indios americanos) }\end{array}$ \\
\hline Edad $\geq 45$ años \\
\hline IFG o IGT identificada previamente \\
\hline Hipertensión $\geq$ 140/90 mmHg \\
\hline HDL colesterol $<35 \mathrm{mg} \%$ y / o triglicéridos $\geq 250 \mathrm{mg} \%$ \\
\hline
\end{tabular}

Historia de diabetes gestacional (DMG) o macrosomía (>4 Kg.)

nadas" durante las ferias de salud en cada EBAIS, dentro de su programación anual, y en el Día Mundial de la Diabetes, como parte de la jornada nacional.

La estrategia selectiva fue desarrollada por medio del apoyo de la Universidad de Costa Rica, buscanda las familias en riesgo (con un diabético en el hogar) y citando a los familiares directos del diabético al EBAIS, para hacerles la prueba de tamizaje por micrométodo con glucómetro. Si el resultado del micrométodo era sugestivo de DM2, se enviaba una glicemia por laboratorio. Se visitó a un 10\% de las familias riesgo. La parte fundamental de búsqueda por tamizaje con micrométodo en la ronda domiciliar por el ATAPS, no se pudo realizar por no disponer de glucómetros. Aun así, a los PRDM se les recomendó hacerse una glicemia consultando en el EBAIS, pero de manera voluntaria, sin sistematización. Como iniciativa propia, en 2 EBAIS, si la persona reunía la condición de PRDM, se entregaron boletas de laboratorio para examen de glicemia en el domicilio durante la ronda domiciliar por el ATAP.

B. ¿A quién se tamizó?: Se recomendó seguir los criterios de la Asociación Americana de Diabetes del año $2000^{17}$, con una modificación: se eliminó la variable etnia hispana como factor de riesgo, al suponer que la mayoría de la población costarricense posee las características que definen esta variable (Cuadro 1).

II. Se revisaron todos los expedientes de los diabéticos registrados por atención primaria a diciembre de 1999 , confirmando sus domicilios y su diagnóstico para tener un registro fiable de ser comparado con los resultados de diciembre de 2000. El porcentaje de cobertura del ASIS de 1999 se calculó con base en el total de pacientes DM2 captados por el ATAP en su ronda domiciliar, o en control en el EBAIS, dividido por el número de pacientes DM2 estimados, considerando una prevalencia de DM2 del 5\% de la población de 20 años y más x 100. Es decir, se consideró como un $100 \%$ de cobertura, la captación del total de los pacientes diabéticos estimados para cada EBAIS, utilizando la estimación general para Costa Rica en el año 2000, que correspondió a un $5 \%$ de la población de 20 y más años (Cuadro 2).

Durante 2000, se anotaron todos los diabéticos captados tanto en control en el EBAIS, como en la ronda domiciliar por el ATAP. Después se comparó la cantidad de pacientes diabéticos de 1999 con los del ASIS de 2000, para valorar el cambio en el porcentaje de cobertura, excluyendo los fallecidos, los traslados antes de diciembre de 1999, los no ubicables y los mal diagnosticados.

\begin{tabular}{|c|c|c|c|c|c|c|c|c|c|}
\hline \multicolumn{10}{|c|}{$\begin{array}{l}\text { Cuadro 2. Personas de } \geq 20 \text { años, número de diabéticos captados en ASIS, número de personas } \\
\text { eliminadas, según causa y porcentaje de cobertura, según EBAIS- } 1999\end{array}$} \\
\hline \multirow[t]{2}{*}{ EBAIS } & \multirow[t]{2}{*}{$\begin{array}{l}\text { Población } \\
320 \text { años }\end{array}$} & \multirow[t]{2}{*}{$\begin{array}{l}\text { DM2 } \\
\text { estimados* }\end{array}$} & \multirow{2}{*}{$\begin{array}{l}\text { DM2 } \\
\text { según } \\
\text { ASIS } \\
1999\end{array}$} & \multicolumn{4}{|c|}{$\begin{array}{l}\text { Personas en registro ASIS eliminadas } \\
\text { según causa }\end{array}$} & \multirow{2}{*}{$\begin{array}{c}\text { Total } \\
\text { captados } \\
1999 \\
\text { después } \\
\text { de revisión }\end{array}$} & \multirow{2}{*}{$\begin{array}{c}\% \\
\text { Cobertura } \\
\text { Nov 1999** }\end{array}$} \\
\hline & & & & $\begin{array}{l}\text { Fallecidos } \\
\text { Mal Dx }\end{array}$ & Traslados & No uk & & & \\
\hline Patarrá & 3220 & 161 & 73 & 1 & 6 & 17 & 0 & 49 & 30.4 \\
\hline Fátima & 2859 & 143 & 108 & 3 & 2 & 8 & 1 & 94 & 65.7 \\
\hline Guido 2 & 2411 & 121 & 66 & 2 & 2 & 6 & 2 & 54 & 44.6 \\
\hline Guido 4 & 2400 & 120 & 88 & 4 & 3 & 11 & 0 & 70 & 58.3 \\
\hline Río Azul & 2285 & 114 & 83 & 0 & 4 & 7 & 0 & 72 & 63.2 \\
\hline Linda Vista & 2652 & 133 & 76 & 5 & 4 & 7 & 0 & 60 & 45.1 \\
\hline TOTAL & 15827 & 791 & 494 & 15 & 21 & 56 & 3 & 399 & 50.4 \\
\hline \multicolumn{10}{|c|}{$\begin{array}{l}\text { * estimados ( } 5 \% \text { población }{ }^{3} 20 \text { años) } \\
\text { ** actualizado después de revisión de I }\end{array}$} \\
\hline
\end{tabular}




\section{Resultados}

\section{Estrategias de tamizaje y captación de nuevos pacientes diabéticos}

Las 4 estrategias no se pudieron implementar sistemáticamente, por tener que respetar la programación anual de los EBAIS. Se captaron en total 174 pacientes diabéticos (Cuadro 3). La oportunística fue la que aportó el mayor número de nuevos diabéticos captados (164 diabéticos) (Cuadro 3). El tamizaje en el nivel de familia de riesgo solo se realizó a 64 PRDM, del 10\% de las casas de familias en riesgo del área estudiada, con una captación de solo un diabético nuevo. Con la visita domiciliar, aunque no se pudo realizar la glicemia por micrométodo, se captaron 6 diabéticos que ya estaban en control en otros centros de salud y 2 que se conocían diabéticos, pero no tenían control alguno. Esto se obtuvo con solo la entrevista que deben realizar los ATAPS en su ronda domiciliar, sobre la presencia de pacientes con enfermedades crónicas. Solo un grupo cautivo de 30 personas adultas mayores de Río Azul se tamizó, sin encontrarse ningún diabético nuevo. Este grupo es tamizado sistemáticamente cada año y ya existían 4 diabéticos conocidos. Durante las jornadas de las ferias de salud y el Día Mundial de la Diabetes se realizaron un promedio de 150 glicemias por micrométodo por EBAIS: la mayoría no en ayunas; se captó únicamente un diabético nuevo.

\section{Calidad del sistema de registro de pacientes diabéti- cos reportados en el ASIS de 1999, de los EBAIS estudiados}

Al revisar los expedientes en 2000, de los pacientes anotados como diabéticos en el ASIS de noviembre 1999, se documentó que 18 habían fallecido (16 antes de nov. 1999), 27 se habían trasladado fuera del área de atracción de los EBAIS (20 antes de nov, 1999), y 56 no se pudieron ubicar; además, 3 no cumplían con los criterios para DM2, es decir un $1 \%$ estaban mal diagnosticados, al ser intolerantes a los carbohidratos y no diabéticos (Cuadro 3).

No había un sistema de actualización de la condición de los pacientes en cuanto a cambio de domicilio, traslado o fallecimiento, existiendo en total 90 diabéticos incluidos en el ASIS de 1999 que no estaban realmente dentro del área de atracción de los EBAIS estudiados para finales de ese año, produciendo un $18.3 \%(90 / 494)$ de sobrerregistro en el ASIS.

\section{Número de diabéticos captados, a diciembre de 2000}

Al 31 de diciembre de 2000 se encontraban registrados 572 DM2 "activos" en el área estudiada, es decir, habían asistido por lo menos a una consulta médica en el año, en algún centro de salud, o fueron documentados como diabéticos en la visita domiciliar de ese año, realizada por ATAP.

\section{Cobertura de atención de la población DM2}

La cobertura de atención en noviembre de 1999, una vez depurado el registro, fue de un $50.4 \%$ con un total de 399 pacientes diabéticos captados. La cobertura a diciembre de 2000 fue de un $72 \%$, un $22 \%$ más que en noviembre de 1999, con un total de 572 pacientes. En algunos EBAIS llegó a ser mayor del 80\% (Cuadro 3).

\section{Discusión}

Este estudio permitió analizar 2 parámetros fundamentales de la atención primaria, para brindar calidad en el tratamiento de enfermedades crónicas, tomando la DM2 como enfermedad prototipo: el diagnóstico temprano y la vigilancia epidemiológica.

\begin{tabular}{|c|c|c|c|c|c|c|c|c|c|}
\hline \multicolumn{10}{|c|}{ Cuadro 3} \\
\hline \multirow[t]{2}{*}{ EBAIS } & \multirow{2}{*}{$\begin{array}{c}\text { DM2 } \\
\text { Estimados } \\
5 \%\end{array}$} & \multirow{2}{*}{$\begin{array}{c}\text { Total } \\
\text { captados } \\
\text { Nov. } 1999^{*}\end{array}$} & \multirow{2}{*}{$\begin{array}{c}\% \\
\text { Cobertura } \\
\text { Nov. 1999* }\end{array}$} & \multicolumn{4}{|c|}{$\begin{array}{c}\text { Nuevos captados según } \\
\text { estrategia }\end{array}$} & \multirow{2}{*}{$\begin{array}{c}\text { Total } \\
\text { captados } \\
\text { Dic. } 2000\end{array}$} & \multirow{2}{*}{$\begin{array}{c}\% \\
\text { Cobertura } \\
\text { Dic. } 2000\end{array}$} \\
\hline & & & & 1 & 2 & 3 & 4 & & \\
\hline Patarra & 161 & 49 & 30.4 & 57 & - & 1 & 1 & 108 & 67.1 \\
\hline Fátima & 143 & 94 & 65.7 & 11 & - & 0 & 1 & 106 & 74.2 \\
\hline Guido 2 & 121 & 54 & 44.6 & 26 & - & 0 & 1 & 81 & 67.2 \\
\hline Guido 4 & 120 & 70 & 58.3 & 30 & - & 0 & 2 & 102 & 85.0 \\
\hline Río Azul & 114 & 72 & 63.2 & 29 & 0 & 0 & 3 & 104 & 91.0 \\
\hline Linda Vista & 133 & 60 & 45.1 & 11 & - & 0 & 0 & 71 & 53.5 \\
\hline Total & 791 & 399 & 50.4 & 164 & 0 & 1 & 8 & 572 & 72.3 \\
\hline
\end{tabular}


Se demostró que el tamizaje en el nivel de atención primaria es efectivo y se pudo aumentar la cobertura de DM2 captados, del $50 \%$ al $72 \%$ en esta Área de Salud, cifra importante de diabéticos que pudieron iniciar su tratamiento oportuno. Esto se logró predominantemente con la estrategia "oportunística", dentro de la atención médica habitual de los EBAIS, sin alterar su programación, con solo enfocar su atención a los PRDM. Por otro lado, las jornadas de tamizaje en ferias y en el Día Mundial de la DM2 no dieron el rendimiento esperado; aunque se tamizaron alrededor de 1000 personas, solo se captó un diabético. Sin embargo, estas jornadas se pueden considerar provechosas como medio para educar a la comunidad sobre esta enfermedad. Con el tamizaje selectivo a familias en riesgo se obtuvo mejor respuesta, ya que con solo tamizar a 64 personas PRDM, se diagnosticó un diabético. Esta estrategia podría ser importante estudiarla de forma más sistemática, ya que en el estudio solo se logró visitar a un $10 \%$ de las familias en riesgo. El tamizaje en visita domiciliar no se pudo evaluar, ya que no se realizó por falta de glucómetros.

En vista de los buenos resultados del tamizaje en este estudio, con solo realizar glicemias a los PRDM que demandaron atención en consulta médica, surge la interrogante: ¿sería probable aumentar esta cifra con un tamizaje sistemático por los ATAPS, tanto en la visita domiciliar como en visita a las familias en riesgo?

Se tratará de contestar a la luz de la bibliografía internacional que considera que cualquier enfermedad sujeta de tamizaje debe contestar 7 preguntas afirmativamente, para que la implementación de este sea válida ${ }^{18}$.

A continuación, se muestra que la Diabetes Mellitus tipo 2 en Costa Rica puede ser sujeta a tamizaje, ya que responde a todas las preguntas en cuestión, afirmativamente.

\section{1. ¿Es una patología con repercusión en la Salud Pública en Costa Rica?}

Esta entidad afecta aproximadamente a 134,000 personas en Costa Rica, de las cuales casi la mitad no lo saben. Es, además, la causante de problemas de visión y ceguera, de insuficiencia renal terminal y de gran parte de las amputaciones de miembros inferiores. Los pacientes consumen una cantidad enorme de recursos médicos del país, ocupando el primer lugar en costos en todos los hospitales (tipos A, B y C) y el cuarto en la consulta externa en adultos en la C.C.S.S ${ }^{3}$.

\section{2. ¿Es la historia natural de la DM2 bien conocida?}

En la bibliografía mundial hay amplia evidencia de su historia natural, en la cual existe un estado preclínico no detectable y luego uno detectable. Usualmente se inicia con una hiperglicemia postprandial como primera alteración y luego se altera la glicemia en ayunas ${ }^{5,8,14}$. Después de un periodo variable de latencia (4-7 años) se desarrollan los síntomas y pasase al estado clínico ${ }^{19}$. Según el control metabólico y la duración de la enfermedad, se pasa al estado de las complicaciones microvasculares que llevarán a la discapacidad o la muerte. En Costa Rica no hay estudios de prevalencia ni de incidencia de complicaciones crónicas, pero los de órdenes internacionales son amplios como para considerar que esta patología tiene un curso similar aquí.

3. ¿Existe en la DM2 un estado preclínico (asintomático) reconocible en el cual la enfermedad pueda ser diagnosticada?

Usando los mismos criterios diagnósticos para individuos sintomáticos, se puede diagnosticar en su estado preclínico, como lo describe por el panel de expertos para el diagnóstico y clasificación de Diabetes Mellitus ${ }^{20}$.

\section{4. ¿El tratamiento precoz y oportuno conlleva a un beneficio superior que cuando el tratamiento se pospone?}

Aunque no existen estudios rigurosos de ensayos comunitarios controlados para evaluar el tamizaje de DM2 en comunidad y su impacto en el nivel internacional, y mucho menos en Costa Rica, está ampliamente reconocido que una atención precoz y de calidad beneficia la reducción de las complicaciones microvasculares como la retinopatía, la nefropatía y la neuropatía ${ }^{21}$. Además, en varios estudios se refiere que la $\mathrm{HbA} 1 \mathrm{c}$ de las personas recién diagnosticadas anda en el orden del $9 \%{ }^{21}$, por lo cual se puede afirmar que cuanto más tempranamente se diagnostique la enfermedad, más pronto podemos iniciar una terapia adecuada y, por consiguiente, mejorar el pronóstico.

\section{5. ¿Son las pruebas diagnósticas que detectan el estado preclínico de la diabetes confiable y aceptable?}

Las pruebas diagnósticas son las mismas para la etapa preclínica y para la clínica. Las pruebas utilizadas en el tamizaje, usualmente bioquímicas, se han desarrollado mucho, con gran sensibilidad y especificidad, como es la del caso la prueba por glucómetro con sangre capilar, tipo sensor $^{22}$. El problema persiste todavía en los rangos de corte para considerara a una persona sospechosa de DM2, para el estado de ayuno o postprandial, con el uso de micrométodos. En cualquier situación, debe tenerse muy claro que una prueba de tamizaje nunca es diagnóstica. Deben seguirse los criterios diagnósticos internacionales. El sistema de salud de Costa Rica ofrece calidad tecnológica para realizar las pruebas diagnósticas certificadas en todo el país, en los laboratorios de la C.C.S.S., y actualmente cuenta con glucómetros tipo sensor en el nivel de atención primaria.

6. ¿Es el costo-beneficio de una intervención de tamizaje en el nivel comunitario favorable? $y$ ¿Existen las facilidades y recursos para tratar los nuevos casos diagnosticados?

La respuesta a la primera parte de esta pregunta no es clara todavía. Existe poca información del costo de los 
tamizajes, según los escenarios en que se aborden. Los tamizajes en el nivel poblacional se han considerado de alto costo y potencialmente ineficientes. El tamizaje selectivo, que tiene como meta subgrupos poblacionales con alta prevalencia de riegos para $\mathrm{DM} 2^{16,17}$, es el que está más recomendado. El tamizaje oportunístico, realizado en los pacientes que asisten a los servicios médicos bueno, ya que las PRDM son fácilmente ubicables y la prueba de tamizaje puede ser en laboratorio, en plasma y en ayunas. Esta última estrategia de abordaje ya está incorporada en las normas de atención integral de la persona adulta en Costa Rica y en el Plan de atención a la salud de las personas 2000-2006 ${ }^{23}$ (PASP). La estrategia selectiva se considera que podría ser efectiva y no de tan alto costo, debido a nuestro modelo de atención primaria, con 871 EBAIS en todo el país.

En cuanto a la segunda parte de la pregunta, el sistema de salud de Costa Rica cubre casi el 95\% de la población y ofrece los tratamientos con hipoglicemiantes y con insulina, que en el estudio "United Kingdom prospecitve diabetes study" (UKPDS) demostraron ser eficientes para el control metabólico $^{21}$. A los pacientes con insulinoterapia, en general también se les facilitan las jeringas, por lo que se puede contestar que sí existen las facilidades y los recursos para tratar los nuevos casos diagnosticados.

\section{7. ¿Tendrá la estrategia de tamizaje continuidad como proceso?}

En la mayoría de los países donde se ha desarrollado, no ha existido continuidad ${ }^{18}$; pero con el modelo de atención primaria en salud, si en Costa Rica, existiera voluntad política, la estrategia podría ser real, por medio del tamizaje domiciliar de PRDM, por los ATAPS.

La estrategia "oportunista" sí es posible que se realice para el año 2006, ya que figura como meta en el PASP $2002-2006^{23}$.

a. Al finalizar 2002 se contará con glucómetros y oftalmoscopios adecuados en toda la red de servicios de la C.C.S.S.

b. Al finalizar 2006, la cobertura mínima del tamizaje por diabetes mellitus será del $70 \%$ de la población identificada en la consulta externa como de alto riesgo.

El otro parámetro por analizar en el estudio, la vigilancia epidemiológica de la DM2, permitió evidenciar que el sistema de registro es deficiente y poco controlado en esta área de salud. La cifra de pacientes DM2 captados, versus los diabéticos estimados en los ASIS de los EBAIS estudiados, presentó un sobrerregistro de 90 personas (18.3\%). La cobertura, según el ASIS de 1999, fue del 62\%, y disminuyó al 50\% una vez depurados los datos contra expedientes (cuadro 3). Esto evidencia que la calidad del sistema registral de pacientes diabéticos no fue adecuada y su actualización no estaba sistematizada (no se excluían los fallecidos, los traslados, cambios de residencia, etc.) Se puede suponer que todavía no existe un sistema de vigilancia (monitoreo) de esta importante enfermedad crónica en el área y probablemente en el país.

Concluimos que Costa Rica puede lograr la detección temprana y tratamiento oportuno de la DM2, por medio de un tamizaje en subgrupos poblacionales de alto riesgo. El esfuerzo ya se está haciendo con la estrategia oportunista y este estudio demuestra que se puede implementar, con poco esfuerzo. Ahora bien, la organización del sistema de salud con base en la atención primaria permitiría desarrollar otra de las estrategias de tamizaje para obtener una mayor cobertura sin alto costo: el tamizaje domiciliar. Logísticamente existen las condiciones para realizarlo, lo que se necesita es llevar a cabo un estudio comunitario controlado para ver su costo-beneficio. Si el resultado de este estudio es favorable, se podría implementar en todo el territorio, como parte de la atención integral de la DM2, convirtiendo al país en pionero en el mundo en la utilización de esta estrategia.

Por otro lado, al contar las áreas de salud con la posibilidad de conocer la cantidad de diabéticos que tienen en control y los captados por los ATAPS, es posible implementar de un sistema de vigilancia de DM tipo 2, que permita evaluar la atención a los diabéticos y a la comunidad en general, incluyendo variables de distinto orden: prevalencia, incidencia, cobertura, mortalidad, morbilidad, complicaciones crónicas, referencias y contrarreferencias, hospitalizaciones, etc. Esto es factible y se tendría un instrumento con qué evaluar la atención en forma dinámica y medir el impacto de las intervenciones programadas alrededor de la patología.

Agradecimiento: Muchas gracias a todo el personal de los 6 EBAIS del Área 3 de Desamparados que participaron en este estudio. Con su trabajo diario y su esfuerzo desinteresado se pudo realizar un tamizaje para DM2 dentro de su programación habitual, mejorando así la cobertura de los diabéticos.

\section{Abstract}

Introduction: Type 2 Diabetes mellitus today must be considered a public health problem in Costa Rica, since it has a great impact in the quality of life of the patients and their families, due to its chronic complications and also in the Social Security System due to its costs. Early diagnosis and prompt treatment decrease the burden of its complications. Therefore, Diabetes screening in a high risk population should be considered beneficial. The purpose of this study was to evaluate the results of a screening intervention in primary care in high risk persons.

Methodology: Screening was implemented along the year 2000 in the adult population with at least one risk factor of 
6 Primary Health Centers, incorporated in the daily routine of the primary health care attention. Four different strategies were used: the "opportunistic" during the regular medical consultation, a selective one during home visits, programmed, directed to captive groups or social organizations, and "Jornadas" during community or national health fairs.

Results: During the year 2000, 173 new diabetic patients were registered by the health centers, either they were newly diagnosed or where known diabetics that lived in the area, but were not following control there. The predominant strategy was the "oportunístic", relaying the responsibility especially upon the physician. The programed strategy in "Jornadas" was undertaken during Health Fairs of the Primary care centers and during the World Diabetes Day, the 14 of November. The selective strategy was not perform systematically since there were no glucometers for the primary health care assistant that does home visits. The proper registration or "coverage" of the diabetic patients of the Health Center Area increased from 50\% in 1999 to $70.2 \%$ in 2000 , considering the former as the percentage of diabetic patients under control or registered divided by the number of diabetics accounted for each health center, considering a prevalence of $5 \%$ of the population 20 years and older.

Conclusion: Costa Rica can accomplish an early detection and prompt treatment of Diabetes mellitus type 2 by screening intervention of high risk individuals. This is already being done with through the "opportunistic" strategy. This study shows that this strategy can be effectively implemented and that it can be operational with little effort. Our Health Care System, based in a primary health care setting, is in position to develop other screening strategies that could give higher coverage with lower costs: such as home visit screening by the primary health assistant. Logistically the conditions are given, however this intervention requires a community study to determine its real cost-benefit. If the results were favorable, such intervention could be implemented as part of diabetes care in the whole country, making Costa Rica a pioneer in the world in this kind of intervention.

\section{Referencias}

1. King H, Aubert R, Herman W. Global burden of Diabetes, 19952025, prevalence, numerical estimates and projections. Diabetes Care 1998; 21:1414-1431.

2. Trejos A, Ortega A. El envejecimiento de la población costarricense. Rev Médica Hosp. Nacional de Niños (Costa Rica) 1985; 20:35-44

3. Morice A, Roselló M, Araúz AG, Sánchez G, Padilla G. Diabetes mellitus en Costa Rica: un análisis interdisciplinario. Capítulo IV: Prevalencia de Diabetes mellitus en Costa Rica. Serie de documentos técnicos. Instituto de Investigación y Enseñanza en nutrición y salud. Costa Rica, agosto 1999.
4. Tan H, Maclean DR. Epidemiology of diabetes mellitus in Canada. Clin Invest Med 1995;18: 240-246.

5. Harris MI, Flegal KM, Cowie CC, Eberhardt MS, Goldstein DE, Little RR, et al. Prevalence of diabetes, impaired fasting glucose, and impaired glucose tolerance in U. S. Adults: the Third National Health and Nutrition Examination Survey 1988-1994. Diabetes Care 1998; 21: $518-524$.

6. Aiello LP, Gardner TW, King GL, Blanken-Ship G, Cavallerano JD, Ferris Fl et al. Diabetic retinopathy (Technical Reviews). Diabetes Care 1998; 21:143-156.

7. Harris MI, Klein R, Cowie CC, Rowland M, Burd-Holt DD. Is the risk of diabetic retinopathy greater in non-Hispanic blacks an Mexican Americans that in non-Hispanic whites with type 2 diabetes, a U.S population Study. Diabetes Care 1998; 21:1230-1235.

8. Rajala U, Laakso M, Qiao Q, Keinanen-Kiukaanniemi S. Prevalence of retinopathy in people with diabetes, impaired glucose tolerance, and normal glucose tolerance. Diabetes Care 1998;21: 1664-1669.

9. Ballard DJ, Humphrey LL, Melton LJ, Frohnert PP, Chu PC, O'Fallon WM et al. Epidemiology of persistent proteinuria in type 2 diabetes mellitus: population-based study in Rochester, Minnesota. Diabetes 1988; 37:405-412.

10. Klein R, Klein BEK, Moss SE. Prevalence of microalbumnuria in older-onset diabetes. Diabetes Care 1993;16: 1325-1330.

11. Lehtinen JM, Uusitupa M, Siitoneno, Pyorala K. Prevalence of neuropathy in newly diagnosed NIDDM and nondiabetic control subjects. Diabetes 1989; 38:1308-1313.

12. Thomas P. Classification, differential diagnosis, and staging of diabetic peripheral neuropathy. Diabetes Care 1997; 46: S54-S57.

13. Turner R, Cull C, Holman R. United Kingdom prospective diabetes study 17: a 9 year update of a randomized, controlled trial on the effect of improved metabolic control on complications in noninsulin-dependent diabetes mellitus. Ann Intern Med 1996; 124:136145.

14. Harris ML. Undiagnosed NIDDM: clinical and public health issues. Diabetes Care 1993;16: 642-652.

15. Harris MI. Sreening for NIDDM: Why is there no national program? Diabetes Care 1994; 17:440-444.

16. Cowie CC, Harris MI, Eberhardt MS. Frecquency and determinants of screening for diabetes in the U. S. Diabetes Care 1994;17: 11581163 .

17. American Diabetes Association: Screening for type 2 diabetes. Diabetes Care 2000; 23 S20-S23.

18. Engelgau M, Venkat K.M, Herman, WH. Sreening for type 2 diabetes, Diabetes Care 2000; 23 1563-1580.

19. Harris MI, Klein R, Welborn Ta,Knuiman MW. Onset of NIDDM occurs at least 4-7 years before diagnosis. Diabetes Care 1992;15: 815-819.

20. Expert committee on the diagnosis and classification of diabetes mellitus. Report of the expert committee on the diagnosis and classification of diabetes mellitus. Diabetes Care 1997;20: 1183-1197.

21. UK Prospective Diabetes Study (UKPDS) group. Intensive blood glucose control with sulphonylureas or insulin compared with conventional treatment and risk of complications in patients with type 2 diabetes. (UKPDS 33) Lancet 1998; 352:837-853.

22. Reney CC, Kirk JK. Performance of three blood glucose meters. Ann Pharmacother 2000; 34:317-21.

23. Caja Costarricense de Seguro Social, Plan de atención a la salud de la personas 2001-2006, pag 100, San José, Costa Rica, 2001 